\title{
Radial mode widths in red giant stars spectra observed by Kepler
}

\author{
Mathieu Vrard ${ }^{1,2, \star}$, Benoît Mosser ${ }^{2,}$, and Caroline Barban², \\ ${ }^{1}$ Instituto de Astrofísica e Ciências do Espaço, Universidade do Porto, CAUP, Rua das Estrelas, 4150-762 Porto, Portugal \\ ${ }^{2}$ LESIA, CNRS, PSL Research University, Université Pierre et Marie Curie, Université Denis Diderot, Observatoire de Paris, \\ 92195 Meudon cedex, France
}

\begin{abstract}
The Kepler space mission has observed many solar-like pulsators, and helped to decipher their fundamental parameters (e.g: mass, radius, rotation). Most of the achievements recently obtained in that domain result from the analysis of the mode frequencies. However, unique information on non-adiabatic physics derives from the height and width of the modes. In this study, we aim at measuring the mode widths of the pressure modes in thousands of Kepler red giants and to analyze their variations in function of stellar parameters. To achieve that, we used a peakbagging technique on the star radial modes. The results show a relation between the radial mode linewidth and the effective temperature of the star as theoretically predicted. We also unveil a clear dependence with mass and stellar evolution for the radial mode width. This means that the mode damping depends on the evolutionary status of the stars.
\end{abstract}

\section{Introduction}

Thanks to the CoRoT [2] and Kepler [8] missions, solarlike oscillations have been detected in thousands of red giants, which enable the precise measurement of their mass and radius [15]. Their oscillation spectra exhibit pressure modes as well as mixed modes [10], allowing the characterization of their internal structure by the measurement of the mode frequencies. Observations enable us to distinguish the stars that have ignited their helium in their core (clump stars) from the ones that burn their hydrogen on a shell (RGB stars) [e.g. 6, 19]. These data also bring constraints on the physical processes occurring in the star interiors. For example, the measurement of the mode width gives access to the mode lifetime. Therefore it allows us to obtain informations on the damping mechanism [e.g. 13]. The previous studies conducted on this subject $[4,18]$ have shown that radial mode widths in red giants behave as in main-sequence stars. However, slight differences arise in the scaling relations between the mode damping and the effective temperature. A dependence with the evolutionary state is also observed. The aim of this work is to confirm and extend the previous results using the most recent data release from the Kepler satellite.

\section{Data analysis method}

\subsection{Data set}

Long-cadence data from Kepler up to the quarter Q17 corresponding to 44 months of photometric observations were used. The red giant sample was selected based on the precise determination of the star seismic parameters and their

\footnotetext{
^e-mail: mathieu.vrard@astro.up.pt
}

evolutionary states performed in [22]. It corresponds to a sample of more than 4700 stars.

\subsection{Measurement of the star radial mode linewidths}

The red giant oscillation spectra exhibit pressure modes and mixed modes [e.g. 5], the latter resulting from the coupling of the acoustic waves propagating inside the stellar envelop and the gravity waves propagating in their core [e.g. 20]. Mixed modes present long lifetime and rapid variations of the mode width which made the characterization of the mode damping difficult [e.g. 9]. As a consequence, we focus our study on radial modes only since they are pure pressure modes.

For each of these stars, a first estimate of the global seismic parameters (the large separation $\Delta v$ and the frequency of maximum oscillation $v_{\max }$ ) has been obtained with the envelope autocorrelation function [16]. The $\Delta v$ values are then refined by the use of the universal pattern [17], which also helps locating the position of the radial modes. The next step was to automatically identify the modes present near the first estimate of the radial mode frequency position given by the universal pattern. In order to do that, we smoothed the power spectra of the light curves. We then locate the nearby local maximas in the smoothed spectrum within a range of frequencies equivalent to a tenth of the large frequency separation around the estimated frequency position of the radial modes. These local peaks are considered to be significant when their heights are above a threshold value corresponding to the rejection of the pure noise hypothesis with a confidence 


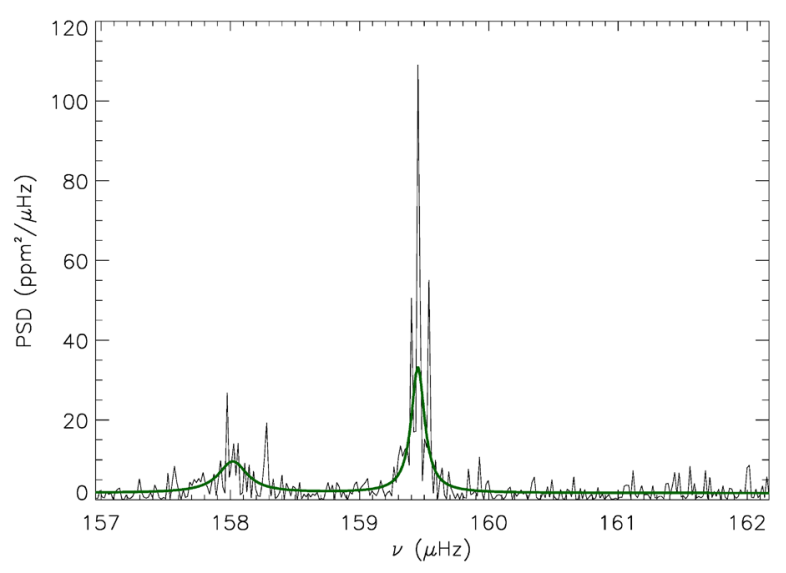

Figure 1: Power density spectrum as a function of frequency for the star KIC3758505 (in black). The fit based on Lorentzian is shown with the solid green line. The peak around $159.5 \mu \mathrm{Hz}$ is a radial mode and the one on the left is the nearby $\ell=2$ mode.

level of $99.9 \%$. Among those modes, the doublet $\ell=0$ and $\ell=2$ can be easily identified by being the modes with the highest height and mode width value.

After that, we fit the radial modes with Lorentzians as well as the nearby modes (like the quadrupolar modes, see Fig. 1) following the MLE technique described in [3] and [21]. The fit is composed of a linear background component and several Lorentzian profiles, one for each of the modes. The fit being local, a complicate modelling of the background is not necessary. This allows us to extract the frequency, height and width of the radial modes.

Individual mode widths $\left(\Gamma_{0}\right)$ were computed following [1] for each of the stars. A mean value of $\Gamma_{0}$ is derived from the weighted average of the width of the three radial modes closest to $v_{\max }$. This way of computing $\Gamma_{0}$ proved to be less sensitive to systematic effects. The individual linewidths are indeed expected to be nearly uniform around $v_{\max }$ as shown by [13]. We also used the seismic scaling relations in order to have an estimate of the stellar masses [15].

\section{Results}

\subsection{Evidence of a mass dependence}

We obtained $\Gamma_{0}$ for more than 4700 stars. We first chose to plot the variation of $\Gamma_{0}$ with the global seismic parameter $\Delta v$ (Fig. 2). It allows us to make appear clear variations of $\Gamma_{0}$ in function of the stellar parameters. We could note that, for stars with similar masses, the radial mode widths in the clump are significantly higher than on the RGB. We also put into light a slight but clear mass dependence, regardless of the evolutionary status. This phenomenon is particularly obvious for helium burning stars. The values of $\Gamma_{0}$ for clump stars show indeed a clear dependence on $\Delta v$ especially in the secondary clump which is a consequence of the mass dependence. Mode widths are directly related to the mode damping. These observa-

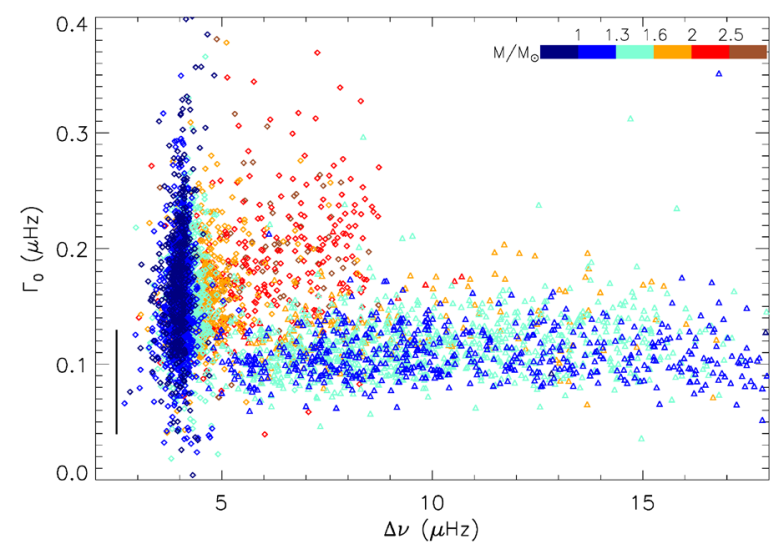

Figure 2: Radial mode width $\left(\Gamma_{0}\right)$ as a function of the large separation $(\Delta v)$. The color code indicates the star masses. The diamonds indicate clump stars and the triangles RGB stars. The black line corresponds to the mean value of $\Gamma_{0}$ uncertainties.

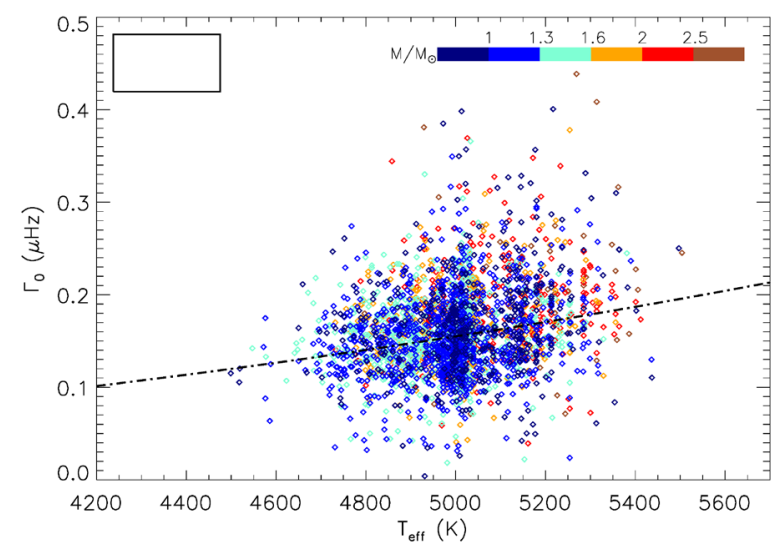

Figure 3: Radial mode width $\left(\Gamma_{0}\right)$ as a function of the stellar effective temperature for clump stars. The color code indicates the stellar masses. The black square in the upper left corner corresponds to the mean value of the uncertainties. The black dasheddotted line represents the width-temperature relation measured for clump stars.

tional results will be used in a further step for assessing the modes damping in red giants.

\subsection{The $\Gamma_{0}-T_{\text {eff }}$ relation}

According to theoretical predictions, the star linewidths should be highly dependent on the stellar effective temperature [7]. However, previous studies have not seen this dependence for red giant stars [4] contrary to subgiants and main-sequence stars. In order to test the theoretical expectations we used the measured effective temperature described in [14] for the Kepler targets. Since we put into light a clear difference of behavior for $\Gamma_{0}$ in function of the stellar evolutionary states, we separated the clump and RGB stars in the following analyzes.

The results for clump stars are shown on Fig. 3, the one for RGB stars on Fig. 4. We found a dependence between 


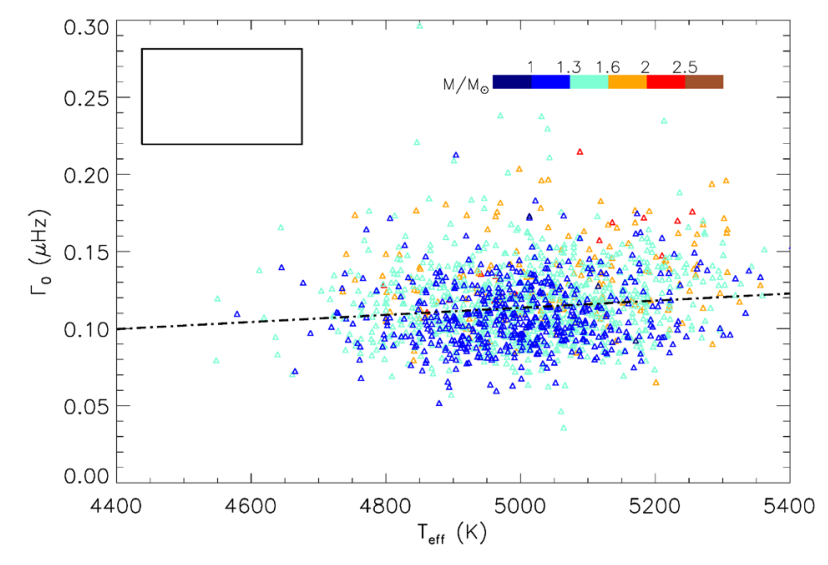

Figure 4: Radial mode width $\left(\Gamma_{0}\right)$ as a function of the stellar effective temperature for RGB stars. The color code indicates the stellar masses. The black square in the upper left corner corresponds to the mean value of the uncertainties. The black dasheddotted line represents the width-temperature relation measured in this work for RGB stars.

the two parameters corresponding to $\Gamma_{0} \propto T_{\mathrm{eff}}^{2.43 \pm 0.21}$ for clump stars and $\Gamma_{0} \propto T_{\text {eff }}^{1.02 \pm 0.22}$ for RGB stars which are non negligible in both cases. For clump stars, the fit can be affected by the presence of high-mass stars with high $T_{\text {eff }}$ but it is not the case for RGB stars. On the RGB, we note a double dependence: first, the radial mode width decreases when $T_{\text {eff }}$ decreases; second, at fixed $T_{\text {eff }}$, the larger the mass, the larger $\Gamma_{0}$. Previous observations did not report a similar $T_{\text {eff }}$ dependence [4]. On the contrary, this kind of $T_{\text {eff }}$ dependence was firmly established for main-sequence and subgiant stars [1, 4]. However, we find a power law with a small exponent, whereas a high value is observed for the other solar-like pulsators, so that our results are far from the ones observed for main-sequence stars.

Finally, the observed relationship significantly differs from the theoretical expectations. The small exponent we find for the power law does not match the high value predicted by the modelling. It follows that our results are far from the expected dependence [7]. On the one side, our sample benefits from a large number of red giants and represents the largest set of data considered up to now for addressing the radial mode widths; as a result, we may consider that our results benefit from a high statistical reliability. On the other side, the data set covers a limited range in effective temperature, so that any possible bias on these $T_{\text {eff }}$ may severely affect the fit.

\section{Conclusions}

In this study, we measured the radial mode width in thousands of red giant stars. Our results emphasize a relationship between the radial mode width and the star effective temperature, which can be modelled with a power law. The exponent is however much below the value observed for subgiants and main-sequence stars. A difference of behavior in function of the stellar evolutionary states was also unveiled, the clump stars having a much higher radial mode width value than RGB stars. We, then, put into light a new mass dependence for the radial mode width present throughout the star evolution. The mode width being directly related to the mode damping, this offers new constraints on this parameter. Theoretical studies about mode damping and excitation suggest that the discovered mass dependence corresponds to theoretical expectations $[11,12]$. However, further theoretical studies are needed to fully exploit the results. Meanwhile, the data analysis will continue, especially on the mode height thus on the oscillation excitation mechanism.

\section{Acknowledgements}

MV acknowledges funding by the Portuguese Science foundation through the grant with reference CIAAUP-03/2016-BPD, in the context of the project FIS/04434, co-funded by FEDER through the program COMPETE.

\section{References}

[1] Appourchaux T. et al., A\&A 537, A134 (2012)

[2] Baglin A. et al., ESA Special Publication 1306, 33 (2006)

[3] Barban C. et al., Astronomische Nachrichten 331, 1016 (2010)

[4] Baudin F. et al., A\&A 529, A84 (2011)

[5] Bedding T. et al., ApJ 713, L176-L181 (2010)

[6] Bedding T. et al., Nature 471, 608-611 (2011)

[7] Belkacem K. et al., A\&A 540, L7 (2012)

[8] Borucki W. J. et al., Science 327, 977 (2010)

[9] Corsaro E. et al., A\&A 579, A83 (2015)

[10] De Ridder J. et al., Nature 459, 398-400 (2009)

[11] Dupret M.-A. et al., A\&A 506, 57-67 (2009)

[12] Grosjean M. et al., A\&A 572, A11 (2014)

[13] Houdek G. et al., A\&A 351, 582-596 (1999)

[14] Huber D. et al., ApJ 211, 2 (2014)

[15] Kallinger T. et al., A\&A 522, A1 (2010)

[16] Mosser B. \& Appourchaux T., A\&A 508, 877-887 (2009)

[17] Mosser B. et al., A\&A 525, L9 (2011)

[18] Mosser B. et al., A\&A 537, A30 (2012)

[19] Mosser B. et al., A\&A 540, A143 (2012)

[20] Unno W. et al., Nonradial oscillations of stars (University of Tokyo Press, Tokyo, 1989) etc

[21] Vrard M. et al., A\&A 579, A84 (2015)

[22] Vrard M. et al., A\&A 588, A87 (2016) 